\title{
Computational Sinc-scheme for extracting analytical solution for the model Kuramoto-Sivashinsky equation
}

\author{
Kamel Al-Khaled, Issam Abu-Irwaq \\ Department of Mathematics and Statistics, Jordan University of Science and Technology, P.O.Box 3030, Irbid 22110, \\ Jordan
}

\begin{tabular}{l}
\hline Article Info \\
\hline Article history: \\
Received Nov 30, 2018 \\
Revised Apr 9, 2019 \\
Accepted Apr 9, 2019 \\
\hline
\end{tabular}

\section{Keywords:}

Kuramoto-sivashinsky equation Sinc-galerkin Sinc-collocation Fixed-point iteration

\begin{abstract}
The present article is designed to supply two different numerical solutions for solving Kuramoto-Sivashinsky equation. We have made an attempt to develop a numerical solution via the use of Sinc-Galerkin method for Kuramoto-Sivashinsky equation, Sinc approximations to both derivatives and indefinite integrals reduce the solution to an explicit system of algebraic equations. The fixed point theory is used to prove the convergence of the proposed methods. For comparison purposes, a combination of a Crank-Nicolson formula in the time direction, with the Sinc-collocation in the space direction is presented, where the derivatives in the space variable are replaced by the necessary matrices to produce a system of algebraic equations. In addition, we present numerical examples and comparisons to support the validity of these proposed methods.
\end{abstract}

Copyright (C) 201x Insitute of Advanced Engineeering and Science. All rights reserved.

\section{Corresponding Author:}

Kamel Al-Khaled,

Jordan University of Science and Technology,

Irbid, P.O.Box 3030, Jordan.

Phone: 00962795010519

Email: kamel@just.edu.jo

\section{INTRODUCTION}

The Kuramoto-Sivashinsky equation (that we abbreviate as K-S), is a simple one-dimensional partial differential equation that exhibiting a particular complex dynamical behavior under some conditions. It arises as an amplitude equation in long-wave, weakly nonlinear stability in a great variety of applications. For example, it arises in concentration waves in chemically reacting systems [1], in flame propagation and reaction combustion [2]. In its simplest form, the equation is given by subject to the initial condition

$$
\begin{gathered}
u_{t}+u u_{x}+\alpha u_{x x}+\beta u_{x x x x}=0, x \in \mathbb{R}, t>0 \\
u(x, 0)=f(x), x \in \mathbb{R}
\end{gathered}
$$

We seek a real-valued function $u=u(x, t)$, defined on $\mathbb{R} \times \mathbb{R}_{0}^{+}$satisfying (1) with $f: \mathbb{R} \rightarrow \mathbb{R}$ is sufficiently smooth function satisfying some decay conditions. The $u_{x x}$ term in (1) is responsible for an instability at large scales, where $\alpha$ is a positive constant; the dissipative $u_{x x x x}$ term provides damping at small scales, and the positive constant $\beta$ playing the role of viscosity; and the non-linear term $u u_{x}$ (which has the same form as that in the Burgers or one-dimensional Navier Stokes equations) stabilizes by transferring energy between large and small scales. The K-S equation, where it models cellular instabilities, pattern formation, turbulence phenomena and transition to chaos. A complete account of the numerical literature on these approximations are mentioned in $[3,4,5,6,7,8]$. Mesh-free methods are the topic of recent research in many areas 
of computational science and approximation theory [9]. Over the past several years mesh-free approximation methods have found their way into many different application areas ranging from engineering applications to the numerical solution of differential equations. A meshless method does not require grid, and only makes use of a set of scattered collocation points. In [10, 11, 12], the authors proposed a mesh-free collocation method and formulate a simple classical radial basis functions for the numerical solution of the $\mathrm{KdV}$ equation, coupled $\mathrm{KdV}$ equations and the K-S equation.

\section{RELATED WORKS}

In recent years various methods have been presented to find approximate solutions for K-S equation. In [13], the quintic B-spline collocation method is implemented to find numerical solution of the KuramotoSivashinsky equation. In [14] a numerical technique based on the finite difference and collocation methods is presented for the generalized Kuramoto-Sivashinsky equation. Chebyshev spectral collocation methods are used in [15] to find approximate solutions for the generalized K-S equation. In [16], authors analyzed and implemented a fully discrete schemes for periodic initial value problems for a general class of dispersively modified Kuramoto-Sivashinsky equations. Time discretizations are constructed using linearly implicit schemes and spectral methods are used for the spatial discretization.

\section{RESEARCH METHOD}

In this paper, we approximate the solution of the K-S equation (1) subject to the initial condition (2) using Sinc-Galerkin method, which builds an approximate solution valid on the entire spatial domain and on a small interval in the time domain. Another benefit of the Sinc methodology is that the scheme presented automatically handles singularities occur at the boundaries with ease. For more details about Sinc solutions of analytic problems with singularties, see [17]. The main idea is to replace derivatives and integrals by their discrete Sinc approximation. Also we solve the K-S solution (1) in the region $a \leq x \leq b, t \geq 0$, by discretizing in time through the use of Crank-Nicolson scheme, and in space by Sinc-collocation method. The layout of the paper is as follows: In section 2, we give the relevant properties of Sinc function such as notations, definitions and some theorem that we need. In section 3, we developed the Sinc-Galerkin method for solving $\mathrm{K}-\mathrm{S}$ equations, and a convergence proof is also given. In section 4, we discuss the mesh-free method together with the Sinc-collocation discretization of the K-S equation. Finally, numerical experiments are presented and some comparisons are made in section 5 . Some concluding remarks are given in the final section.

\section{PROPOSED METHOD}

The goal of this section is to recall notations and definitions of the Sinc function that will be used in this paper. These are discussed in [3, 4], and mainly, we will recall section 2 of $[6,18,19]$. The Sinc function is defined on the whole real line $\mathbb{R}$ by

$$
\operatorname{sinc}(x)=\frac{\sin (\pi x)}{\pi x}, x \in \mathbb{R}
$$

\subsection{Preliminaries}

Recall that a radial basis function is a function whose value depends only on the distance of its input to a central point. For a series of nodes equally spaced $h$ apart, the Sinc function can be written as a radial basis function:

$$
S(j, h)(k h)=\delta_{j k}^{(0)}=\left\{\begin{array}{c}
1, k=j \\
0, k \neq j
\end{array}\right.
$$

Let $\delta_{k j}^{(-1)}=\frac{1}{2}+\delta_{k j}$, where

$$
\delta_{k j}=\int_{0}^{k-j} \frac{\sin (\pi t)}{\pi t} d t
$$

We define a matrix $I^{(-1)}$ whose $(k, j)$ th entry is given by $\delta_{k j}^{(-1)}$. If a function $f(x)$ is defined on the real line, then for $h>0$, the series 


$$
C(f, h)(x)=\sum_{j=-\infty}^{\infty} f(j h) \operatorname{sinc}\left(\frac{x-j h}{h}\right), j=0, \mp 1, \ldots
$$

is called the Whittaker cardinal expansion, which has been extensively studies in [4, 3]. In practice, we need to use a finite number of terms in the above series, say $j=-N, \ldots, N$, where $N$ is the number of sinc grid points. For a restricted class of functions known as the Paly-Weiner class, which are entire functions, the sinc interpolation and quadrature formulae are exact [4]. A less restricted class of functions that are analytic only on an infinite strip containing the real line, and that allow specific growth restrictions has exponentially decaying absolute errors in the sinc approximation.

Definition 1 Let $\mathcal{D}_{d}$ denote the infinite strip domain of width $2 d, d>0$, given by

$$
\mathcal{D}_{d}=\{w=u+i v:|v|<d \leq \pi / 2\}
$$

To construct approximation on the interval $\Gamma=\left(0, T_{0}\right)$, which is our time interval in this paper, we consider the conformal map $\Upsilon(t)=\ln \left(\frac{t}{T_{0}-t}\right)$, the map $\Upsilon$ carries the eye-shaped region

$$
\mathcal{D}=\left\{z=x+i y:\left|\arg \left(\frac{z}{T_{0}-z}\right)\right|<d \leq \pi / 2\right\}
$$

onto the infinite strip $\mathcal{D}_{d}$. For the sinc method, the basis functions on the interval $\Gamma$ at $z \in \mathcal{D}$ are derived from the composite translated sinc functions

$$
S_{j}(z)=S(j, h) \circ \Upsilon(z)=\operatorname{sinc}\left(\frac{\Upsilon(z)-j h}{h}\right) .
$$

The function $z=\Upsilon^{-1}(w)=\frac{T_{0} \exp (w)}{1+\exp (w)}$ is an inverse mapping of the $w=\Upsilon$. We define the range of $\Upsilon^{-1}$ on the real line as

$$
\Gamma=\left\{\Upsilon^{-1}(y) \in \mathcal{D}:-\infty<y<\infty\right\}=\left(0, T_{0}\right)
$$

The sinc grid points $z_{k} \in \Gamma$ in $\mathcal{D}$ will be denoted by $t_{k}$, because they are real, and is given by

$$
t_{k}=\Upsilon^{-1}(k h)=\frac{T_{0} \exp (k h)}{1+\exp (k h)}, k=0, \mp 1, \mp 2, \ldots
$$

To construct an approximation in the interval $(-\infty, \infty)$, which is our space domain in this part, we replace $\Upsilon$ by $\phi(x)=x$. To further explain of the sinc method, an important class of functions is denoted by $\mathbf{L}_{\alpha}(\mathcal{D})$. The properties of the functions in $\mathbf{L}_{\alpha}(\mathcal{D})$ and detailed discussion are given in [4]. We recall the following definition followed by two Theorems for our purpose.

Definition 2 Let $\mathbf{L}_{\alpha}(\mathcal{D})$ be the class of all analytic functions $f$ in $\mathcal{D}$, for which there is a number $C_{0}$ such that, for $\rho(z)=\exp (\Upsilon(z))$, we have

$$
|f(z)| \leq C_{0} \frac{|\rho(z)|^{\alpha}}{[1+|\rho(z)|]^{2 \alpha}}, \forall z \in \mathcal{D}
$$

If $x$ is on the arc $\Gamma$, we obtain the following theorem

Theorem 1 Let $f(x) \in \mathbf{L}_{\alpha}(\mathcal{D})$, $\alpha$ a positive constant, then taking $h_{x}=\sqrt{\pi d /\left(\alpha N_{x}\right)}$ it follows that

$$
\sup _{x \in \Gamma}\left|f^{(n)}(x)-\sum_{j=-N_{x}}^{N_{x}} f\left(x_{j}\right) S_{j}^{(n)}(x)\right| \leq C_{1} N_{x}^{\frac{n+1}{2}} \exp \left(-\sqrt{\pi d \alpha N_{x}}\right)
$$

for $n=0,1, \ldots, m$ with $C_{1}$ a constant independent of $N_{x}$. 
In the next Theorem, we shall give a general formula for approximating the integral $\int_{a}^{\nu} F(u) d u, \nu \in \Gamma$. To this end, we state the following result, which we will use to approximate the obtained integral equation.

Theorem 2 Let $\frac{F(t)}{\Upsilon^{\prime}(t)} \in \mathbf{L}_{\alpha}(\mathcal{D})$, with $0<\alpha \leq 1, \delta_{j k}^{(-1)}$ be defined as above, $N_{t}$ be positive integer, and $h_{t}$ be selected as $h_{t}=\sqrt{\pi d /\left(\alpha N_{t}\right)}$, then there exists a positive constant $C_{2}$ independent of $N_{t}$, such that

$$
\left|\int_{a}^{t_{k}} F(t) d t-h_{t} \sum_{j=-N_{t}}^{N_{t}} \delta_{j k}^{(-1)} \frac{F\left(t_{k}\right)}{\Upsilon^{\prime}\left(t_{k}\right)}\right| \leq C_{2} \exp \left(-\sqrt{\pi d \alpha N_{t}}\right)
$$

The sinc method requires that the derivatives of sinc functions be evaluated at the nodes. Technical calculations provide the following results that will be useful in formulating the discrete system [4, 3], and these quantities are delineated by

$$
\begin{gathered}
\delta_{j k}^{(m)}=\left.h^{m} \frac{d^{m}}{d x^{m}}\left[S_{j} \circ \phi(x)\right]\right|_{x=x_{k}}, \text { where } \\
\delta_{j k}^{(0)}=\left\{\begin{array}{l}
1, j=k \\
0, j \neq k,
\end{array} \quad \delta_{j k}^{(2)}=\left\{\begin{array}{l}
\frac{-\pi^{2}}{3}, j=k \\
\frac{-2(-1)^{k-j}}{(k-j)^{2}}, j \neq k
\end{array} \quad \text { and, } \quad \delta_{j k}^{(4)}=\left\{\begin{array}{l}
\frac{\pi^{4}}{5}, j=k \\
\frac{4(-1)^{k-j}\left[6-(k-j-1)^{2} \pi^{2}\right]}{(k-j-1)^{4}}, j \neq k
\end{array}\right.\right.\right.
\end{gathered}
$$

Then we define the $m \times m$ Toeplitz matrices $I^{(q)}, q=0,1,2,4$ whose $j k-t h$ entry is $\delta_{j k}^{(q)}, q=0,1,2,4$. The matrix $I^{(0)}$ is the identity matrix.

Also we define the diagonal matrix $\mathcal{D}(g(x))=\operatorname{diag}\left[g\left(x_{-N}\right), \ldots, g\left(x_{0}\right), \ldots, g\left(x_{N}\right)\right]^{T}$.

\subsection{Implementation of the method}

The objective of this section is to construct a solution to the K-S equation using the Sinc-Galerkin method. Integrate equation (1) in section 2 with respect to $t$, we get the Volterra integral equation

$$
u(x, t)=-\int_{0}^{t}\left(u(x, \tau) u_{x}(x, \tau)+\alpha u_{x x}(x, \tau)+\beta u_{x x x x}(x, \tau)\right) d \tau+f(x)
$$

with the assumption that the initial condition $f(x) \in \mathbf{L}_{\alpha}(\mathcal{D})$. To obtain direct discretization of equation (5), and since our domain is $(x, t) \in \mathbb{R} \times\left(0, T_{0}\right)$, the relevant maps are defined as follows. In the space direction, we choose $\phi(x)=x$, which maps the infinite strip $\mathcal{D}_{d}$ onto itself. In the time direction, we choose the map $\Upsilon(t)=\ln \left(t /\left(T_{0}-t\right)\right)$ that carries the region $\mathcal{D}$ onto $\mathcal{D}_{d}$. Define the basis elements for $(-\infty, \infty),\left(0, T_{0}\right)$ to be $S\left(m, h_{x}\right) \circ \phi(x), m=-N_{x}, \ldots, N_{x}, S\left(k, h_{t}\right) \circ \Upsilon(t), k=-N_{t}, \ldots, N_{t}$, respectively. The mesh sizes $h_{x}$ and $h_{t}$ represent the mesh sizes in the infinite strip $\mathcal{D}_{d}$ for the uniform grid $\left\{i h_{x}\right\},-\infty<i<\infty$ and $\left\{j h_{t}\right\},-\infty<j<\infty$. The Sinc grid points $x_{i} \in(-\infty, \infty)$ in $\mathcal{D}_{d}$ and $t_{j} \in\left(0, T_{0}\right)$ in $\mathcal{D}$ are inverse images of equispaced grid points, i.e., $x_{i}=\phi^{-1}\left(i h_{x}\right)=i h_{x}$ and $t_{j}=\Upsilon^{-1}\left(j h_{t}\right)=\frac{T_{0} \exp \left(j h_{t}\right)}{1+\exp \left(j h_{t}\right)}$. In equation (5), we carry out Sinc approximations of $u_{x}(x, t), u_{x x}(x, t)$ and $u_{x x x x}(x, t)$; to proceed, we use Theorem 1 , and the replacement of the derivatives with respect to $x$ by its approximation yields

$$
u_{x}(x, t) \approx\left(\frac{-1}{h_{x}} I_{m_{x}}^{(1)}\right) u\left(x_{i}, t\right), \quad u_{x x}(x, t) \approx\left(\frac{1}{h_{x}^{2}} I_{m_{x}}^{(2)}\right) u\left(x_{i}, t\right), \quad \text { and } u_{x x x x}(x, t) \approx\left(\frac{1}{h_{x}^{4}} I_{m_{x}}^{(4)}\right) u\left(x_{i}, t\right)
$$

where $m_{x}=2 N_{x}+1$. In equation (5), evaluating all functions at the $x$-nodes, and replacing the derivative by its appropriate approximation, we obtain the Volterra integral equation

$$
\mathbf{u}(t)=-\int_{0}^{t}\left(\mathbf{u}(\tau) A_{1} \mathbf{u}(\tau)+\alpha A_{2} \mathbf{u}(\tau)+\beta A_{4} \mathbf{u}(\tau)\right) d \tau+\mathbf{f}^{0}
$$

where $A_{1}=\frac{-1}{h_{x}} I_{m_{x}}^{(1)}, A_{2}=\frac{1}{h_{x}^{2}} I_{m_{x}}^{(2)}$, and 


$$
A_{4}=\frac{1}{h_{x}^{4}} I_{m_{x}}^{(4)}
$$

with $\mathbf{u}(t)=\left(u_{-N_{x}}, \ldots, u_{N_{x}}\right)^{T}$, where $u_{i}(t)=u\left(x_{i}, t\right)$ and $\mathbf{f}^{0}=\left(f\left(z_{-N_{x}}\right), \ldots, f\left(x_{N_{x}}\right)\right.$. We next collocate with respect to the $t$ variable via the use of Theorem 2, with the matrix $B=h_{t} I_{m_{t}}^{(-1)} \mathcal{D}\left(\frac{1}{\Upsilon^{\prime}}\right), m_{t}=2 N_{t}+1$ and the nodes $t_{j}=\Upsilon^{-1}\left(j h_{t}\right)$ for $j=-N_{t}, \ldots, N_{t}$. Define the matrix $F^{0}=\left[f\left(x_{i}, 0\right)\right]$. Then the solution of equation (6) in matrix form is given by the rectangular $m_{x} \times m_{t}$ matrix $U=\left[u_{i j}\right]$,

$$
U=-\left(U \circ A_{1} U+\alpha A_{2} U+\beta A_{4} U\right) B^{T}+F^{0}
$$

where the notation $\circ$ denotes the Hadamard matrix multiplication. Note that in our discretization, we are taking the time nodes as rows and the space nodes as columns, so the matrix $\left(U \circ A_{1} U+\alpha A_{2} U+\beta A_{4} U\right)$ forms the vector nodes for the integral in (6). In equation (8) the vector $F^{0}$ has the same dimensions as the vector $U$, and every column of $F^{0}$ consists of the same vector $f^{0}$.

To solve the system in equation (8), one idea is to produce a sequence of iterations that converges to the exact solution of the K-S equation. Equation (8) can be written as

$$
U=G(U)+F^{0}
$$

where $G(U)=-\left(U \circ A_{1} U+\alpha A_{2} U+\beta A_{4} U\right) B^{T}$. By selecting an initial approximation $U^{0}$, we iterate the continuous map $G$ repeatedly via the formula

$$
U^{(n+1)}=G\left(U^{(n)}\right)+F^{0}, n=0,1,2, \ldots
$$

\subsection{Validation of Sinc-Galerkin method}

Throughout this subsection, we use the notation $U=\left[u\left(x_{i}, t_{j}\right)\right]$ to denote the $m_{x} \times m_{t}$ matrix of node values of the function $u(x, t)$, and so on for other functions. Set, $K(x, t)=-\int_{0}^{t} M(\tau) d \tau$, where $M(\tau)=\left(u(x, \tau) u_{x}(x, \tau)+\alpha u_{x x}(x, \tau)+\beta u_{x x x x}(x, \tau)\right)$. Using Theorem 2 , the approximation of the integral in matrix form has an error as

$$
\left\|\left[K\left(x_{i}, t_{j}\right)\right]-B U\right\| \leq C_{2} \exp \left(-\sqrt{\pi d \alpha N_{t}}\right)
$$

In the next Theorem, we show that our approximation produce an error of exponential order

Theorem 3 Let $u(x, t)$ be the exact solution of the $K-S$ equation, and let $U$ be the matrix defined as in (9). Then for $N_{x}, N_{t}>\frac{16}{\pi d \alpha}$, there is a constant $C$ independent of $N_{x}, N_{t}$ such that

$$
\sup _{\left(x_{i}, t_{j}\right)}\|u(x, t)-U\| \leq C N^{2} \exp (-\sqrt{\pi d \alpha N}) \text {, where } N=\min \left\{N_{x}, N_{t}\right\}
$$

Proof: Evaluate the integral equation (5) at the nodes $\left(x_{i}, t_{j}\right)$ where $i=-N_{x}, \ldots, N_{x}, j=-N_{t}, \ldots, N_{t}$, we get

$$
u\left(x_{i}, t_{j}\right)=-\int_{0}^{t_{j}}\left(u\left(x_{i}, \tau\right) u_{x}\left(x_{i}, \tau\right)+\alpha u_{x x}\left(x_{i}, \tau\right)+\beta u_{x x x x}\left(x_{i}, \tau\right)\right) d \tau+f\left(x_{i}\right)
$$

To approximate the above integral, we use the definite integral formula Theorem 2 , and define a matrix $B=$ $h_{t} I_{m_{t}}^{(-1)} \mathcal{D}\left(\frac{1}{\Upsilon^{\prime}}\right)$, with $m_{t}=2 N_{t}+1$, we obtain

$$
U=-\left(U \circ U_{x}+\alpha U_{x x}+\beta U_{x x x x}\right) B^{T}+F^{0}+C_{2} \exp \left(-\sqrt{\pi d \alpha N_{t}}\right) .
$$

With the use of the approximations mentioned in Theorem 1, we obtain

Error $=U+\left(U \circ A_{1} U+\alpha A_{2} U+\beta A_{4} U\right) B^{T}-F^{0}=C_{2} \exp \left(-\sqrt{\pi d \alpha N_{t}}\right)+C_{12} N_{x}^{2} B^{T} \exp \left(-\sqrt{\pi d \alpha N_{x}}\right)$. 
But from the definition of the matrix $B$, and since $\frac{1}{\Upsilon^{\prime}(t)}=\frac{t\left(T_{0}-t\right)}{T_{0}}$, which has its maximum at $T_{0} / 4$, therefore, $B=T \tilde{B}$. It is known that $\left\|I_{m_{t}}^{(-1)}\right\| \leq 1.1$, (see, [4]), so the matrix $B$ can be written as $B=T_{0} \tilde{B}$ where each entry in the matrix $\tilde{B}$ is bounded by $1.1 h_{t} T_{0} / 4$. With all of these bounds, the error term can be bounded as

$$
\text { Error }=U-\left(-U \circ A_{1} U-\alpha A_{2} U-\beta A_{4} U\right) B^{T}-F^{0} \leq C N^{2} \exp (-\sqrt{\pi d \alpha N})
$$

for some constant $C$. Finally choose $N=\min \left\{N_{x}, N_{t}\right\}$, and notice that the function $N^{2} \exp (-\sqrt{\pi d \alpha N})$ is decreasing when $N>\frac{16}{\pi d \alpha N}$.

\subsection{Fixed-point iteration}

We now take up the existence proof of the solution of the discrete system by fixed-point iteration. The idea is to produce a sequence that converge to the solution of the K-S equation. By selecting an initial approximation $U^{0}$ we iterate the continuous map $G$ repeatedly via the formula

$$
U^{n+1}=G\left(U^{n}\right)+F^{0}, \quad n=0,1,2, \ldots
$$

Since $G\left(U^{n}\right)=-\left(U^{n} \circ A_{1} U^{n}+\alpha A_{2} U^{n}+\beta A_{4} U^{n}\right) B^{T}$, where $B=T_{0} \tilde{B}$ for some bounded matrix $\tilde{B}$. Choose $T_{0}$ sufficiently small such that $\|G(U)\| \leq k$ and $\|d G(U)\|<k$, for any $U$ in any given fixed ball $\mathcal{B}$ about the origin, where $k$ is a constant with $0<k<1$. Now

$$
\begin{aligned}
\left\|U^{n+1}-U^{n}\right\| & =\left\|G\left(U^{n}\right)-G\left(U^{n-1}\right)\right\| \\
& \leq k\left\|U^{n}-U^{n-1}\right\| \\
& \vdots \\
& \leq k^{n}\left\|U^{1}-U^{0}\right\|
\end{aligned}
$$

which implies that

$$
\begin{aligned}
\left\|U^{n+1}-U^{0}\right\| & \leq k^{n}\left\|U^{1}-U^{0}\right\|+k^{n-1}\left\|U^{1}-U^{0}\right\|+\ldots+\left\|U^{1}-U^{0}\right\| \\
& \leq \frac{1}{1-k}\left\|U^{1}-U^{0}\right\|
\end{aligned}
$$

and for positive integer $p$, we have

$$
\left\|U^{n+p}-U^{n}\right\| \leq \frac{k^{p}}{1-k}\left\|U^{1}-U^{0}\right\|
$$

so, with a choice of $k \in(0,1)$ and $U^{0}, U^{1}, \ldots$, we see that all iterates will remain in the ball

$$
\mathcal{B}=\left\{V:\|V\| \leq \frac{1}{1-k}\left\|U^{1}-U^{0}\right\|\right\}
$$

Also there is an integer $N$ such that $\left\|U^{n+p}-U^{n}\right\|<\epsilon$ for all $n>N$, and for any $p$. Therefore the sequence $\left\{U^{n}\right\}$ is a Cauchy sequence, and hence converges to some $U^{*}$ where $\lim _{n \rightarrow \infty} U^{n}=U^{*}$. For uniqueness, suppose there are two distinct solutions, say $U^{*}$ and $U^{\star}$, then using $\|d G(U)\|<k$, we have

$$
\left\|U^{\star}-U^{*}\right\|=\left\|G\left(U^{\star}\right)-G\left(U^{*}\right)\right\| \leq k\left\|U^{\star}-U^{*}\right\|
$$

for $k=1 / 2$, we arrive at a contradiction, this shows that the solution is unique. With the notation as above we have proved the Theorem.

Theorem 4 Given a constant $R>0$, there is a constant $T_{0}>0$ such that if $\left\|U^{1}-U^{0}\right\|<R / 2$, then the solution (12) has a unique solution. Moreover, the iteration scheme (13) with $U^{0}=0$ converges to this unique solution. 


\subsection{Validation of Mesh-Free Sinc-collocation method} conditions

Consider the nonlinear K-S equation in (1), subject to the initial condition (2), and the boundary

$$
u(a, t)=0, u(b, t)=0, u_{x}(a, t)=0, u_{x}(b, x)=0, t>0 .
$$

To implement the Sinc-collocation method, following [20], we discretize time derivative of the nonlinear K-S equation using the Crank-Nicolson scheme, and space derivatives by the $\theta$-weighted $(\theta=1 / 2)$ scheme successive two time levels $n$ and $n+1$

$$
\left(\frac{u^{n+1}-u^{n}}{\delta t}\right)+\left(\frac{\left(u u_{x}\right)^{n+1}+\left(u u_{x}\right)^{n}}{2}\right)+\alpha\left(\frac{\left(u_{x x}\right)^{n+1}+\left(u_{x x}\right)^{n}}{2}\right)+\beta\left(\frac{\left(u_{x x x x}\right)^{n+1}+\left(u_{x x x x}\right)^{n}}{2}\right)=0
$$

where $u^{n}=u\left(x, t^{n}\right)$ is the value of the solution at the $n$-th time step, and $t^{n}=t^{n-1}+\delta t$, where $\delta t$ is a time step size. The nonlinear term $\left(u u_{x}\right)^{n+1}$ must be linearized before continuing. This can be accomplished by using the following formula which obtained by applying the Taylor expansion, as follows

$$
\left(u_{x}\right)^{n+1} \approx\left(u_{x}\right)^{n}+\delta t \frac{u_{x}^{n+1}-u_{x}^{n}}{\delta t}+\mathcal{O}\left(\delta t^{2}\right),\left(u u_{x}\right)^{n+1} \approx\left(u u_{x}\right)^{n}+\delta t\left[\left(u_{t}\right)^{n} u_{x}^{n}+(u)^{n} u_{x t}^{n}\right]+\mathcal{O}\left(\delta t^{2}\right)
$$

which can be simplified to

$$
\left(u u_{x}\right)^{n+1} \approx\left(u u_{x}\right)^{n}+\delta t\left[u_{x}^{n} \frac{(u)^{n+1}-(u)^{n}}{\delta t}+u^{n} \frac{u_{x}^{n+1}-u_{x}^{n}}{\delta t}\right]+\mathcal{O}\left(\delta t^{2}\right)
$$

Finally, we arrive at the linearization

$$
\left(u u_{x}\right)^{n+1} \approx(u)^{n+1} u_{x}^{n}+u_{x}^{n+1} u^{n}-u^{n} u_{x}^{n}
$$

So equation (15) can be rewritten as

$$
\left(\frac{u^{n+1}-u^{n}}{\delta t}\right)+\left(\frac{\left.u^{n} u_{x}^{n+1}+u^{n+1} u_{x}^{n}\right)}{2}\right)+\alpha\left(\frac{\left(u_{x x}\right)^{n+1}+\left(u_{x x}\right)^{n}}{2}\right)+\beta\left(\frac{\left(u_{x x x x}\right)^{n+1}+\left(u_{x x x x}\right)^{n}}{2}\right)=0
$$

Rearranging equation (18), we get

$$
u^{n+1}+\frac{\delta t}{2}\left(u^{n} u_{x}^{n+1}+u^{n+1} u_{x}^{n}+\alpha\left(u_{x x}\right)^{n+1}+\beta\left(u_{x x x x}\right)^{n+1}\right)=u^{n}-\frac{\delta t}{2}\left(\alpha\left(u_{x x}\right)^{n}+\beta\left(u_{x x x x}\right)^{n}\right)
$$

where $u^{n}$ are the $n$-th iterates of the approximate solutions. Now the space variable is discretized upon the use of Sinc-collocation at the points

$$
\left\{x_{1}=a, \ldots, x_{i}+a+(i-1) h, \ldots, x_{N}=b\right\}, \quad h=\frac{|b-a|}{N-1}
$$

The solution of equation (15) is interpolated and approximated by means of the Sinc functions as

$$
u^{n}(x)=\sum_{j=0}^{N} u_{j}^{n} S_{j}(x), S_{j}(x)=\operatorname{sinc}\left(\frac{x-(j-1) h-a}{h}\right)
$$

The unknown parameters $u_{j}$ in equation (21) are to be determined by collocation method. Therefore, for each collocation point $x_{i}$ in (20), equation (21) can be written as

$$
u^{n}\left(x_{i}\right)=\sum_{j=0}^{N} u_{j}^{n} S_{j}\left(x_{i}\right), i=1, \ldots, N .
$$

Substituting equation (22) into equation (18), for the interior points $x_{i}, i=1, \ldots, N-1$, we get 


$$
\begin{aligned}
& \sum_{j=0}^{N} u_{j}^{n+1} S_{j}\left(x_{i}\right)+\frac{\delta t}{2}\left[\sum_{j=0}^{N} u_{j}^{n} S_{j}\left(x_{i}\right) \sum_{j=0}^{N} u_{j}^{n+1} S_{j}^{\prime}\left(x_{i}\right)+\right. \\
& \left.\sum_{j=0}^{N} u_{j}^{n+1} S_{j}\left(x_{i}\right) \sum_{j=0}^{N} u_{j}^{n} S_{j}^{\prime}\left(x_{i}\right)+\alpha \sum_{j=0}^{N} u_{j}^{n+1} S_{j}^{\prime \prime}\left(x_{i}\right)+\beta \sum_{j=0}^{N} u_{j}^{n+1} S_{j}^{\prime \prime \prime \prime}\left(x_{i}\right)\right] \\
& =\sum_{j=0}^{N} u_{j}^{n} S_{j}\left(x_{i}\right)-\frac{\delta t}{2}\left[\alpha \sum_{j=0}^{N} u_{j}^{n} S_{j}^{\prime \prime}\left(x_{i}\right)+\beta \sum_{j=0}^{N} u_{j}^{n} S_{j}^{\prime \prime \prime \prime}\left(x_{i}\right)\right]
\end{aligned}
$$

The boundary conditions reads as

$$
\sum_{j=0}^{N} u_{j}^{n+1} S_{j}\left(x_{0}\right)=\sum_{j=0}^{N} u_{j}^{n+1} S_{j}^{\prime}\left(x_{0}\right)=0, \sum_{j=0}^{N} u_{j}^{n+1} S_{j}\left(x_{N}\right)=\sum_{j=0}^{N} u_{j}^{n+1} S_{j}^{\prime}\left(x_{N}\right)=0 .
$$

The system (23) and (24) contain $N+1$ equations with $N+1$ unknowns $u_{j}^{n}$ which can be easily solved by Gaussian elimination method. Once the values of $u_{j}^{n}$ are obtained then the solution for $u$ can be derived from equation (21). Now we switch to a matrix representation of Equations (23) and (24). Define the matrices

$$
\left\{N_{1}^{n}\right\}_{i j}=\left[\sum_{\ell=0}^{N} \delta_{\ell}^{(0)} u_{\ell}^{n}\left(x_{i}\right)\right] \delta_{j}^{(1)}\left(x_{i}\right),\left\{N_{2}^{n}\right\}_{i j}=\left[\sum_{\ell=0}^{N} \delta_{\ell}^{(1)} u_{\ell}^{n}\left(x_{i}\right)\right] \delta_{j}^{(0)}\left(x_{i}\right)
$$

The above two equations are valid for $i=1, \ldots, N-2$, and in the rows $1, N-1$, and $N$ the boundary conditions (24) hold true. So in matrix form equations (23), (24) can be written as

$$
\left[I^{(0)}+\frac{\delta t}{2}\left(N_{1}^{n}+N_{2}^{n}\right)+\frac{\delta t}{2}\left(\alpha I^{(2)}+\beta I^{(4)}\right)\right] u^{n+1}=\left[I^{(0)}-\frac{\delta t}{2}\left(\alpha I^{(2)}+\beta I^{(4)}\right)\right] u^{n}+F^{n+1}
$$

If the condition of the left hand side of equation (25) is small, then $u^{n}$ for any time $t_{k}$ can be easily calculated from the initial condition. For stability analysis of the Sinc-collocation solution, the evolution of error can be written as

$$
\left[I^{(0)}+\frac{\delta t}{2}\left(N_{1}^{n}+N_{2}^{n}\right)+\frac{\delta t}{2}\left(\alpha I^{(2)}+\beta I^{(4)}\right)\right] e^{n+1}=\left[I^{(0)}-\frac{\delta t}{2}\left(\alpha I^{(2)}+\beta I^{(4)}\right)\right] e^{n}
$$

where $e^{n}=\left|u_{\text {exact }}^{n}-u_{\text {approx }}^{n}\right|$, where $u_{\text {exact }}^{n}$ and $u_{\text {approx }}^{n}$ are the exact and Sinc-collocation approximated solutions at time $t^{k}$ respectively. Equation (26) can be written as

$$
e^{n+1}=M^{-1} N e^{n}
$$

where

$$
M^{-1} N=\left[I^{(0)}+\frac{\delta t}{2}\left(N_{1}^{n}+N_{2}^{n}\right)+\frac{\delta t}{2}\left(\alpha I^{(2)}+\beta I^{(4)}\right)\right]^{-1}\left[I^{(0)}-\frac{\delta t}{2}\left(\alpha I^{(2)}+\beta I^{(4)}\right)\right] .
$$

The scheme is considered numerically stable if $\rho\left(M^{-1} N\right) \leq 1$, where $\rho($.$) denoted the spectral radius. Stability$ is assured if

$$
\frac{1-0.5 \delta t\left(\alpha \lambda_{2}+\beta \lambda_{4}\right)}{1+0.5 \delta t\left(\lambda_{N_{1}}+\lambda_{N_{2}}\right)+0.5 \delta t\left(\alpha \lambda_{2}+\beta \lambda_{4}\right)} \leq 1
$$

where $\lambda_{2}, \lambda_{4}, \lambda_{N_{1}}, \lambda_{N_{2}}$ are the eigenvalues for the matrices $I^{(2)}, I^{(4)}, N_{1}^{n}, N_{2}^{n}$ respectively. In order to study stability of Sinc methods, we should find some bound for the eigenvalues of the matrices appeared in Equation (28). For a bound for the Toeplitz matrices $I^{(2)}$ and $I^{(4)}$, we refer the reader to [17] where well-known results for upper and lower bounds are established. While for other two matrices $N_{1}$ and $N_{2}$, the eigenvalues depends on the choice of the parameter $N$, that is already taken into account for the scheme. The stability of the scheme 
and conditioning of the component matrices of the matrix $M^{-1} N$ depend on the weight parameter and the minimum distance between any two collocation points $h$ in the domain set $[a, b]$.

Remark I: In the previous sections, we showed how to replace the derivatives and integrals by the necessary matrices if the boundary conditions are homogeneous. In particular, the Sinc methodology presented in this paper is still applicable to equation (1) with non-homogeneous boundary conditions. The non-homogeneous boundary conditions can be transformed to homogenous boundary conditions by the change of variables $w(x, t)=u(x, t)-\chi(x, t)$, where $\chi(x, t)$ is an analytic function that is defined as in Lemma 5.1 of [21].

\section{RESULTS AND ANALYSIS}

Choosing examples with known solutions allows for a more complete error analysis. In order to assess the advantages of the proposed methods, Sinc-Galerkin method over the mesh-free Sinc-collocation method in terms of accuracy and efficiency for solving K-S equation, we have applied the two methods to two different examples. For the numerical results:

Sinc-Galerkin method(SGM): We apply the Sinc-Galerkin method which has the matrix form (9), In all calculations, we have used $d=\frac{\pi}{2}, \alpha=\frac{1}{2}, N_{x}=N_{t}=64$, and the step-sizes $h_{x}, h_{t}$ can be determined by $h_{x}=\sqrt{\pi d /\left(\alpha N_{x}\right)}, h_{t}=\sqrt{\pi d /\left(\alpha N_{t}\right)}$. One advantages of the Sinc method is that it automatically determines the graded mesh.

Mesh-free Sinc-collocationn method(SCM): We also solve the K-S equation using the mesh-free Sinccollocation method (25). In our computational work, we take time step sizes $\delta t=0.05$ through the interval $[-5,5]$ and $N=100$ for the set of collocation points as in equation (20). The step-size $h$ is the minimum distance between any two points in equation (20). The computations associated with the two examples were performed using Mathematica.

Example 1 Consider the equation

$$
u_{t}+u u_{x}+2 u_{x x}+u_{x x x x}=0
$$

Where we set $\alpha=2$ and $\beta=1$ into equation (1). This problem has exact solution [14]

$$
u(x, t)=-\frac{1}{\kappa}+\frac{60}{19} \kappa\left(-38 \kappa^{2}+2\right) \tanh \theta+120 \kappa^{3} \tanh ^{3} \theta
$$

where $\theta=\kappa x+t$ and $\kappa=\frac{1}{2} \sqrt{22 / 19}$. We will use this solution, evaluated at $t=0$, as the initial condition, also we extract the required boundary conditions from the exact solution on the interval $[-10,10]$.

From the numerical results in Table 1, it can be seen that the approximate solution (using either method) is quite close to the exact solution. This shows the approximate solution is efficiency. A surface plot of the numerical solution is shown in Figure 1 using Sinc-Galerkin method.

Table 1. Comparison results for Example 1

\begin{tabular}{ccccc}
\hline$t$ & $x$ & Exact & Sinc-Galerkin & Mesh-Free-Collocation \\
\hline 0.25 & -6 & -5.04757 & -5.04758 & -5.04758 \\
& -4 & -3.58451 & -3.58448 & -3.58449 \\
& -2 & 2.68033 & 2.68962 & 2.68963 \\
& 0 & -5.32919 & -5.32005 & -5.32010 \\
& 2 & -2.90157 & -2.90110 & -2.90113 \\
& 4 & 0.89126 & 0.891233 & 0.891232 \\
0.5 & 6 & 1.46202 & 1.46202 & 1.46202 \\
& -6 & -4.91406 & -4.91410 & -4.91408 \\
& -4 & -2.64236 & -2.64271 & -2.64246 \\
& -2 & 3.46494 & 3.46144 & 3.46140 \\
& 0 & -7.08063 & -7.08060 & -7.08061 \\
& 2 & -1.4381 & -1.43859 & -1.43862 \\
& 4 & 1.14153 & 1.14138 & 1.14128 \\
& 6 & 1.49241 & 1.49252 & 1.49250 \\
\hline
\end{tabular}




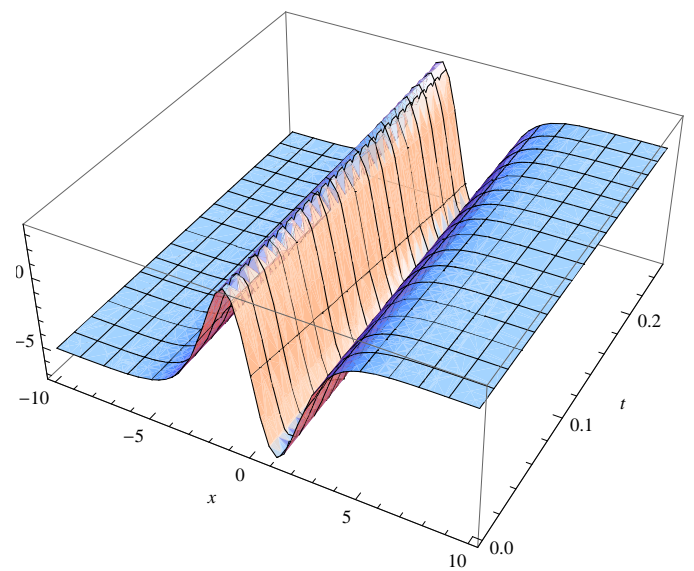

Figure 1. The Sinc-Galerkin solution on $-10<x<10,0<t<0.25$, for Example 1

Remark II: We have shown in Theorem 1 that the problem has a local solution in the interval $(0, T)$ provided that $T$ is sufficiently small. It might be possible that the scheme will diverge for large $T$. To run the scheme, we find a smaller time interval say $\left(0, T_{1}\right)$, in which the scheme will converge, and solving Equation (1) using the given initial condition (2). Then we find a $T_{2}>0$ and solve the system over the interval $\left(T_{1}, T_{2}\right)$, where the initial condition now is the solution found in the interval $\left(0, T_{1}\right)$ evaluated at $t=T_{1}$. This means that the system so far has a solution in the interval $\left(0, T_{2}\right)$. Continuing in this way, we generate a sequence $T_{1}, T_{2}, T_{3}$, to get for (1) defined for all $0<t<T$ such that $T_{1} \leq T_{2} \leq T_{3} \leq \ldots \leq T$.

Example 2 As a second example, we consider equation (1) with $\alpha=\beta=1$, subject to the Gaussian initial condition

$$
u(x, 0)=\exp \left(-x^{2}\right)
$$

with boundary conditions

$$
u(-5, t)=0, u(5, t)=0, u_{x}(5, t)=0, u_{x}(5, x)=0, \quad t>0 .
$$

The K-S equation subject to the Gaussian initial condition (30) exhibiting the chaotic behavior over a finite spatial domain. The numerical results are presented in Figures 2 and 3. A surface plot of the numerical solution is shown in Figure 4 using Sinc-Galerkin method, and Figure 5 using Mesh-free Sinc-collocation.

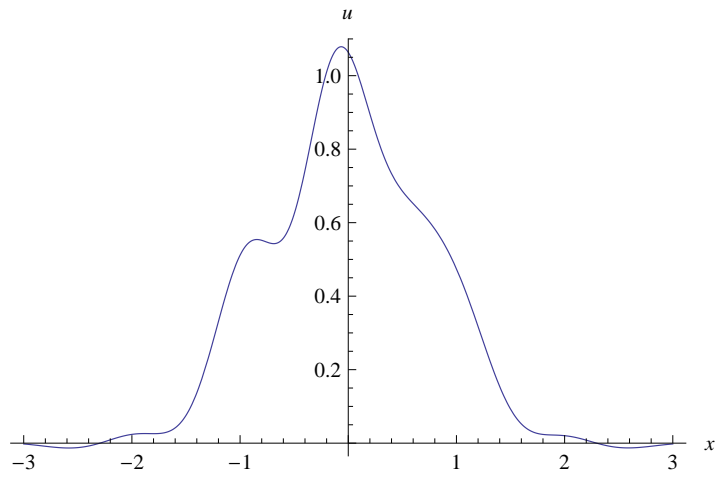

(a)

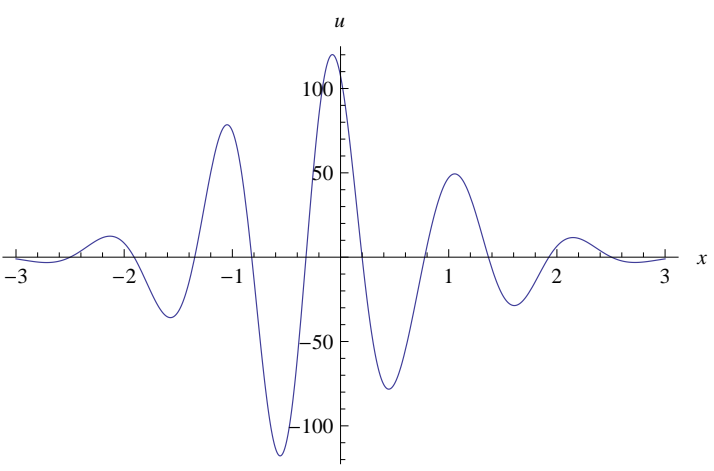

(b)

Figure 2. (a) The chaotic solution with Gaussian initial condition at $t=1$ for Example 2 by Sinc-Galerkin, (b) The chaotic solution with Gaussian initial condition at $t=3$ for Example 2 by Mesh-Free, with $N=160$ 


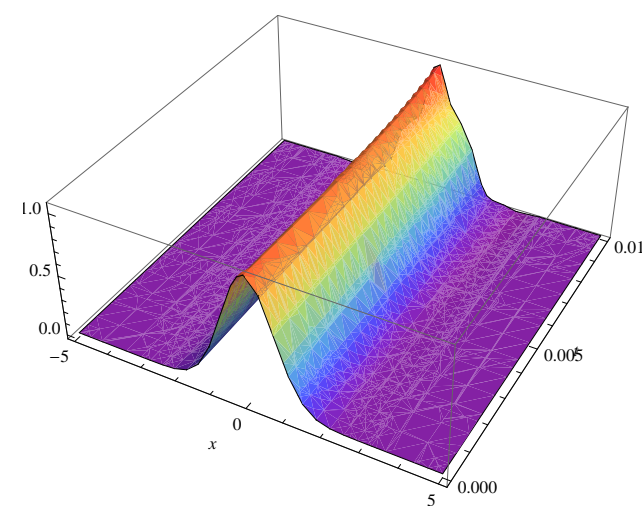

(a)

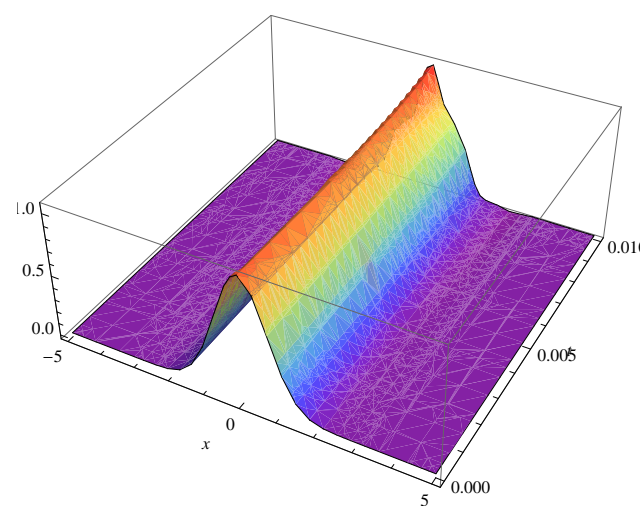

(b)

Figure 3. (a) The solution $u(x, t)$ Sinc-Galerkin method $N_{x}=64, N_{t}=64, T_{0}=1$ for Example 2, (b) The solution $u(x, t)$ using Mesh-free Sinc-collocation method $N=160$ for Example 2

\section{CONCLUSION}

The fundamental goal of this paper is to propose efficient algorithms for solving the K-S equation. Both the Sinc-Galerkin method and Mesh-free Sinc-collocation method were described in details, and implemented to compute a numerical solution to the K-S equation. A convergence proof was provided for the Sinc-Galerkin, and a brief stability analysis was provided for the mesh-free Sinc-collocation method, which produced a necessary condition for stability of the method. The efficiency of the methods was tested on two examples. The results obtained by both methods are very close to analytical ones. The algorithms were found to be stable, exponentially convergent in space and reliable numerical method for solving the K-S equation. The numerical results illustrate that the two methods are equivalent theoretically.

\section{REFERENCES}

[1] Y. Kuramoto, "Diffusion-induced chaos in reaction systems," Suppl. Prog. Theor. Phys., Vol. 64 (1978), Pages 346-367.

[2] G.I. Sirashinsky, "On flame propagation under conditions of stoichiometry," SIAM J. Appl. Math., Vol. 39 (1980), Pages 67-82.

[3] J. Lund, K.L. Bowers, "Sinc methods for quadrature and differential equations," SIAM, Philadelphia, (1992).

[4] F. Stenger, "Numerical Methods Based on Sinc and Analytic Functions," Springer-Verlag, New York, (1993).

[5] Kamel Al-Khaled, "Numerical study of Fisher's reaction-diffusion equation by the Sinc collocation method," J. Comput. Appl. Math., Vol. 137 (2001), Pages 245-255.

[6] Marwan Alquran, Kamel Al-Khaled, "Sinc and solitary wave solutions to the generalized BenjaminBona-Mahony-Burgers equations," Phys. Scr., Vol. 83 (2011) 065010(6pp).

[7] Kamel Al-Khaled, Mohammed Al-Refai, Ameen Alawneh, "Traveling wave solutions using He's variational method and the tanh method for nonlinear coupled equations," Appl. Math. Comput., Vol. 202 (2008), Pages 233-242.

[8] Akbar Mohebbi, Zahra Faraz, "Solitary wave solution of nonlinear Benjaminonaahonyurgers equation using a high-order difference scheme," Computational and Applied Mathematics. Vol. 36 (2),(2017), Pages 915-927

[9] Reza Mokhtari, Maryam Mohammadi, "Numerical solution of GRLW equation using Sinc-collocation method," Comput. Phys. Commun., Vol. 181 (2010), Pages 1266-1274.

[10] Sirajul Haq, Siraj-ul-Islam, Marjan Uddin, "A meshfree interpolation method for the numerical solution of KdV-Burgers equation.” Appl. Math. Model., Vol. 33(8) (2009), Pages 3442-3449.

[11] Siraj-ul-Islam, Sirajul Haq, Marjan Uddin, "A meshfree interpolation method for the numerical solution 
of the coupled nonlinear partial differential equations," Engineering Analysis with Boundary Elements, Vol. 33(3) (2009), Pages 399-409.

[12] Mehri Sajjadian, "The shock profile wave propagation of Kuramoto-Sivashinsky equation and solitonic solutions of generalized Kuramoto-Sivashinsky equation," Universitatis Apulensis, Vol. 38 (2014) pages 163-176.

[13] R.C. Mittal, Geeta Arora, "Quintic B-spline collocation method for numerical solution of the KuramotoSivashinsky equation,” Comm. Nonlinear Sci. Numer. Simulat., Vol. 15 (2010), Pages 2798-2808.

[14] Mehrdad Lakestani, Mehdi Dehgan, "Numerical solutions of the generalized Kuramoto-Sivashinsky equation using B-spline functions," Appl. Math. Model., Vol. 36 (2012), Pages 605-617.

[15] A.H. Khader, R.S. Temsah, "Numerical solutions of the generalized Kuramoto-Sivashinsky equation by Chebyshev collocation method," Comput. Math. with Applic., Vol. 56 (2008), Pages 1465-1472.

[16] G. Akrivis, D.T. Papageorgiou, Y.S. Smyrlis, "Computational study of the dispersively modified Kuramoto-Sivashinsky equation," SIAM J. SCI. COMPUT., Vol. 34(2) (2012), pages 792-813.

[17] F. Stenger, "Handbook of Sinc Numerical Methods," Boca Raton, FL: CRC Press, (2010).

[18] Marwan Alquran, Kamel Al-Khaled, Tridip Sardar, Joydev Chattopadhyay, "Revisited Fisher's Equation in a new outlook: A fractional derivative approach," Physica A., Volume 438 (2015), Pages 81-93.

[19] Marwan Alquran, Kamel Al-Khaled, "Approximations of Sturm-Liouville eigenvalues using sincGalerkin and differential transform methods," Applications and Applied Mathematics, Volume 5(1) (2010), Pages 128-147.

[20] Marjan Uddin, Sirajul Haq, Siraj-ul-Islam, "A mesh-free numerical method for solution of the family of Kuramoto-Sivashinsky equations," Appl. Math. Comput., Vol. 212 (2009), Pages 458-469.

[21] M. El-Gamel, A. Mohsen, A.A. El-Mohsen, "Sinc-Galerkin method for solving biharmonic problems," Appl. Math. Comput., Vol. 247 (2014), Pages 386-396.

\section{BIOGRAPHIES OF AUTHORS}

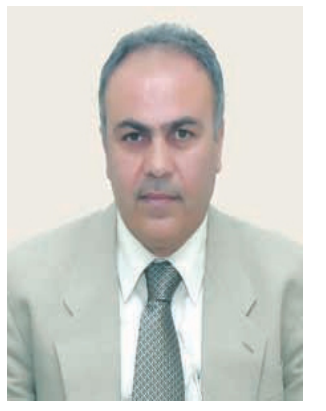

Kamel Al-Khaled is Full Professor, Department of Mathematics and Statistics, Faculty of Science and Arts, Jordan University of Science and Technology, Irbid 22110, Jordan. email: kamel@just.edu.jo

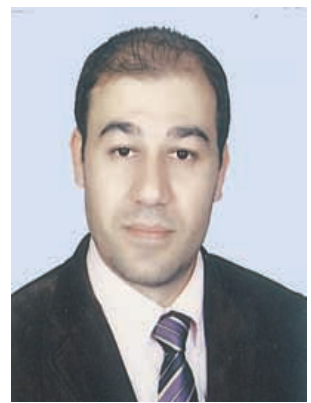

Issam Abu-Irwaq is Instructor, Department of Mathematics and Statistics, Faculty of Science and Arts, Jordan University of Science and Technology, Irbid 22110, Jordan. email: imabuirwaq@just.edu.jo 